\title{
Probabilistic Assessment of the Process-Noise Covariance Matrix of Discrete Kalman Filter State Estimation of Active Distribution Networks
}

\author{
L. Zanni, S. Sarri, M. Pignati, R. Cherkaoui and M. Paolone \\ École Polytechnique Fédérale de Lausanne (EPFL) \\ Lausanne, Switzerland \\ lorenzo.zanni@epfl.ch
}

\begin{abstract}
The accuracy of state estimators using the Kalman Filter (KF) is largely influenced by the measurement and the process noise covariance matrices. The former can be directly inferred from the available measurement devices whilst the latter needs to be assessed, as a function of the process model, in order to maximize the KF performances. In this paper we present different approaches that allow assessing the optimal values of the elements composing the process noise covariance matrix within the context of the State Estimation (SE) of Active Distribution Networks (ADNs). In particular, the paper considers a linear SE process based on the availability of synchrophasors measurements. The assessment of the process noise covariance matrix, related to a process model represented by the ARIMA $[0,1,0]$ one, is based either on the knowledge of the probabilistic behavior of nodal network injections/absorptions or on the aposteriori knowledge of the estimated states and their accuracies. Numerical simulations demonstrating the improvements of the KF-SE accuracy achieved by using the calculated matrix $Q$ are included in the paper. A comparison with the Weighted Least Squares (WLS) method is also given for validation purposes.
\end{abstract}

Keywords-Active distribution networks; Kalman filter; probabilistic assessment; process noise covariance matrix; real-time state estimation; phasor measurement unit

\section{INTRODUCTION}

One of the main challenging tasks related to the operation of Active Distribution Networks (ADNs) is the development of accurate and fast (i.e. sub-second) State Estimation (SE) processes (e.g., [1], [2]). In what follows we make reference to a SE process that uses time-tagged measurements available with high refresh rate (typically those streamed by Phasor Measurement Units - PMUs - with rates of tens of frames per second [3], [4]). Indeed, all the functionalities associated to the Real-Time (RT) operation of ADNs like: (i) optimal voltage control, (ii) feeders congestion management, (iii) losses minimization, (iv) fault detection and location, (v) post-fault management, and (vi) increase of the system reliability [5]-[8], might be largely improved if the knowledge of the network state is available with high accuracy and refresh rate (e.g., [9], [10]).

A well-known approach used for the estimation of power networks state relies on the use of the Kalman Filter (KF) (e.g. [11]-[17]). As known, compared to traditional Weighted Least Square (WLS) method (e.g., [18]), it accounts the available

The research leading to the results presented in this paper has received funding from the NanoTera Swiss National Science Foundation project $S^{3}$ Grids and from the European Community's FP7-ICT-2011-8 under the grant agreement $n^{\circ} 318708(C-D A X)$. measurements and the time-varying nature of the process to be identified by means of a suitable-defined process model that predicts the system state in advance. As a consequence, the KF is a two-stage algorithm. The first 'prediction part' projects the previous time step state forward in time by means of a predefined process model. The second 'estimation part' corrects the predicted state by accounting the available measurements and the accuracies of both process model and measurements.

In this respect, one of the key factors that largely influence the KF accuracy is the knowledge of two error covariance matrices: (i) the so-called process noise covariance matrix, and (ii) the measurement noise covariance matrix.

As discussed in [19], if both noise covariance matrices are not properly defined, the robustness of the KF algorithm is not necessarily satisfied. Additionally, in [17] the relative influence of these two uncertainties has been discussed with reference to the $\mathrm{SE}$ of ADNs performed by using the Iterative KF (IKF) process.

Concerning the measurement noise covariances, they represent the accuracies of the measurement devices and can be easily inferred. On the other hand, the process noise covariances represent the uncertainties introduced by the process model to predict the next system state. It is worth observing that, in general, in the literature dealing with power systems SE using the KF, the values of the process noise covariance matrix are arbitrarily selected although, in principle, they need to be computed if the process is known (e.g., [20], [21]). Therefore, an appropriate assessment of this matrix is of fundamental importance for the maximization of the KF-SE accuracy and represents the objective of this paper.

Within this framework, the Authors of [22] have summarized and discussed some of the novel methods proposed in the last decade for the estimation of the noise covariance matrix for non-linear state estimators. In [23], the Authors have analyzed the tuning of the process and noise covariance matrices in order to optimize the performance of a fault detection process based on the Extended KF (EKF). More recently, in [13] it has been presented a two-stage KF for power systems SE: in the first stage an adaptive KF algorithm identifies and corrects the impact of incorrect system modeling and/or bad PMU measurements; in the second stage the estimated bus voltages are fed into an EKF to obtain the dynamic states. 
Within the framework of KF-based SE of power systems, this paper proposes a probabilistic assessment of the process noise covariance matrix of a Discrete Kalman Filter (DKF) algorithm. The algorithm uses a linear formulation of the DKF in which nodal phasor measurements, composed by bus voltages and nodal injected/absorbed currents, are supposed to be provided by PMUs. In this respect, three methods are proposed: the first one relies on the knowledge of the probability distributions of the nodal absorbed/injected powers deduced from historical measurements; then, the assessment of the process noise covariance matrix is performed by means of a probabilistic load flow using the Monte Carlo method. The second one uses a sliding time window of the previouslyestimated states to directly infer time-varying cumulative distributions of the process noise covariance matrix. The third method uses the uncertainties of the estimated states represented by the a-posteriori estimate error covariance matrix to update the process noise covariance matrix.

The assessment of the performances of the proposed methods, with respect to a DKF-SE process applied to the case of the IEEE 13-bus distribution test feeder [24], is discussed by comparing the performances of the filter with those obtained with a-priori selected values of the process noise covariance matrix. Besides, a further comparison with the SE results provided by a Linear WLS (LWLS) method proposed in [2] is also given.

The structure of the paper is the following: Section II focuses on the description of the DKF-SE. Section III shows the influence of the process noise covariance matrix on the DKF-SE accuracy. Section IV describes the formulation of the three proposed methods that allows assessing the optimal process noise covariance matrix; it also presents the simulation results and the validation of the methods. Section V provides the final remarks and the application fields.

\section{THE ADOPTED DisCRETE KALMAN FILTER}

This section provides the analytical formulation of the linear DKF-based power system SE algorithm including measurements coming from PMUs.

In general, $\mathrm{KF}$ addresses the problem of estimating the state $\mathbf{x} \in \mathfrak{R}^{s}$ (where $\mathfrak{R}^{s}$ is the $s$-dimensional field of real numbers) of a discrete-time system process governed by the following set of linear stochastic equations (e.g., [11], [12]):

$$
\begin{gathered}
\mathbf{x}_{k}=\mathbf{A} \mathbf{x}_{k-1}+\mathbf{B} \mathbf{u}_{k-1}+\mathbf{w}_{k-1} \\
\mathbf{z}_{k}=\mathbf{H} \mathbf{x}_{k}+\mathbf{v}_{k}
\end{gathered}
$$

where:

- $\quad \mathbf{x}_{k} \in \mathfrak{R}^{s}$ and $\mathbf{x}_{k-1} \in \mathfrak{R}^{s}$ represent the system state at the current time step $k$ and at the previous time step $k-1$, respectively. The state of a three-phase (3-ph) power system is commonly represented by the nodal voltage phasors of the three phases at each network bus.

- $\quad \mathbf{u}_{k-1} \in \mathfrak{R}^{l}$ represents a set of control variables at time step $k-1$. In the power system domain, these variables can be nodal injected powers and/or currents as well as line flows represented by powers and/or currents.

- $\mathbf{w}_{k-1} \in \mathfrak{R}^{s}$ represents the process noise at time step $k-1$, assumed to be white and with a normal probability distribution $p(\mathbf{w}) \sim N(0, \mathbf{Q})$ where $\mathbf{Q}$ is the so-called process noise covariance matrix.

- A is a $s^{\times} s$ matrix that links the system state at the current time step $k$ with the state at the previous time step $k-1$. Matrix A might change at each time step;

- B is a $s \times l$ matrix that relates the system state to the control variables at time step $k-1$.

- $\quad \mathbf{z}_{k} \in \mathfrak{R}^{m}$ represents the set of measurements at time step $k^{1}$.

- $\quad \mathbf{v}_{k} \in \mathfrak{R}^{m}$ represents the measurement noise at time step $k$, assumed to be white and with a normal probability distribution $p(\mathbf{v}) \sim N(0, \mathbf{R})$. $\mathbf{R}$ is the so-called measurement noise covariance matrix. The measurement noise is also assumed to be independent from the process noise.

- $\quad \mathbf{H}$ is a $m \times s$ matrix that relates the measurements set and the system state, both referred to the current time step $k$. In case $\mathbf{z}_{k}$ is represented by nodal injected powers, the function that links the measurements with the system state is nonlinear. While using only nodal voltage and injected current phasors measurements, the above-mentioned function becomes linear if (1) ad (2) are written in rectangular coordinates.

Since the paper is targeting ADNs, it is worth reminding that the peculiar characteristics of these networks (e.g., high resistance to reactance $\mathrm{R} / \mathrm{X}$ ratio, high level of imbalance of lines, loads, and Distributed Generators - DGs -) require the adoption of 3-phase unbalanced SE processes. Moreover, the DKF-SE adopted by the Authors relies only on measurements provided by PMUs that, as above-mentioned, enable to obtain a measurement Jacobian matrix $\mathbf{H}$ (which is composed by the partial derivatives of the measurements as a function of the system state) consisting of constant elements, namely: zeros, ones, and elements of the 3-ph compound admittance matrix of the network. In this respect, the state of a power system of $n$ buses can be expressed in rectangular coordinates as follows:

$$
\begin{aligned}
& \mathbf{x}=\left[V_{r}^{1 a}, V_{r}^{1 b}, V_{r}^{1 c}, \ldots, V_{r}^{n a}, V_{r}^{n b}, V_{r}^{n c},\right. \\
& \left.V_{i}^{1 a}, V_{i}^{1 b}, V_{i}^{1 c}, \ldots, V_{i}^{n a}, V_{i}^{n b}, V_{i}^{n c}\right]
\end{aligned}
$$

where:

- $\quad a, b$, and $c$ are the three phases;

- $V_{r}$ and $V_{i}$ are the real and imaginary part of the voltage phasors, respectively;

In view of the above, the size $s$ of the system state vector $\mathbf{x}$ is $n \cdot 3 \cdot 2$. Moreover, the DKF equations can be divided in two parts: (i) the prediction equations responsible for projecting forward in time the previous state $\mathbf{x}_{k-1}$ in order to obtain an apriori estimate $\mathbf{x}_{k}{ }_{k}$ of the state for the current time step, and (ii) the estimation equations incorporating the measurements into the a-priori estimate providing an improved a-posteriori estimate $\mathbf{x}_{k}$.

\section{1) Prediction equations}

$$
\begin{gathered}
\mathbf{x}_{k}^{-}=\mathbf{x}_{k-1} \\
\mathbf{P}_{k}^{-}=\mathbf{P}_{k-1}+\mathbf{Q}_{k}
\end{gathered}
$$

${ }^{1}$ It is worth reminding that the number of measurements should be equal or larger than the number of system state variables in order to preserve the network observability (e.g. [18]). However, the observability analysis is beyond the scope of this paper. 


\section{2) Estimation equations}

$$
\begin{gathered}
\mathbf{K}_{k}=\mathbf{P}_{k}^{-} \mathbf{H}^{T}\left(\mathbf{H} \mathbf{P}_{k}^{-} \mathbf{H}^{T}+\mathbf{R}\right)^{-1} \\
\mathbf{x}_{k}=\mathbf{x}_{k}^{-}+\mathbf{K}_{k}\left[\mathbf{z}_{k}-\mathbf{H} \mathbf{x}_{k}^{-}\right] \\
\mathbf{P}_{k}=\left(\mathbf{I}-\mathbf{K}_{k} \mathbf{H}\right) \mathbf{P}_{k}^{-}
\end{gathered}
$$

where:

- $\mathbf{P}^{-}$is a $s^{\times} s$ matrix representing the a-priori estimate error covariances at time step $k$;

- $\mathbf{P}_{\mathrm{k}-1}$ and $\mathbf{P}_{\mathrm{k}}$ represent the a-posteriori estimate error covariance matrices at time step $k-1$ and $k$, respectively;

- $\quad \mathbf{K}_{\mathrm{k}}$ is a $s \times m$ matrix whose expression is chosen to minimize the a-posteriori estimate error covariances. It is the socalled 'Kalman gain' or 'blending factor'.

In our case, (4) represents the process model assumed to be the ARIMA model of order $(0,1,0)$ (i.e., it is composed by the process (1) in which $\mathbf{A}$ is constant and equal to the identity matrix $\mathbf{I}$, and $\mathbf{B}$ is a null matrix). In other words, the predicted state at the current time step $k$ is supposed to be equal to the estimated state at the previous time step $k-1$.

\section{ON THE INFLUENCE OF THE PROCESS NOISE COVARIANCE MATRIX ON THE ACCURACY OF THE KALMAN FILTER STATE ESTIMATION}

In general, the matrices $\mathbf{Q}$ and $\mathbf{R}$ might change at each time step. $\mathbf{R}$ can be easily defined since it represents the accuracies of the measurement devices (therefore, it does not change along the time). Whereas $\mathbf{Q}$, associated to the probabilistic distributions of $\mathbf{w}_{k-1}$ of (1), changes, in general, at each time step. In this respect, before proposing the optimal assessment of $\mathbf{Q}_{k}$, this section shows the influence of different $\mathbf{Q}$, assumed to be constant along the time, on the accuracy of the adopted DKF-SE process. Furthermore, a comparison with the LWLS method is provided.

The power network that has been used to obtain the SE results presented in this paper is the IEEE 13-bus distribution test feeder [24] shown in Fig. 1. Compared to what defined in [24], the network is assumed with a $15 \mathrm{kV}$ rated voltage. The lines are composed by the unbalanced ones corresponding to the \#602 conductors configuration of [24]. The loads/DGs are characterized by unbalanced power absorptions/injections, respectively. Bus 1 represents the connection to a subtransmission network characterized by the short circuit power $S_{s c}=300 \mathrm{MVA}$ and a ratio between real and imaginary parts of the short circuit impedance $R_{s c} / X_{s c}=0.1$.

The power profiles of the loads and DGs used in the simulations are shown in Fig. 2. They come from real measurements taken in a real distribution network located in the South-West region of Switzerland. In particular, the data refer to two types of loads, commercial and residential, and two types of DGs, a photovoltaic installation and a mini-hydro power plant. In what follows, we have adopted a time window of 20s for which measurements from PMUs each $20 \mathrm{~ms}$ (i.e., with streaming rate of 50 frames-per-second) are assumed to be available. The assumed PMU location is given in Fig. 1 and has been chosen to achieve the full network observability. They measure both the nodal voltage phasors and the injected/absorbed current phasors of the loads and/or DG units.

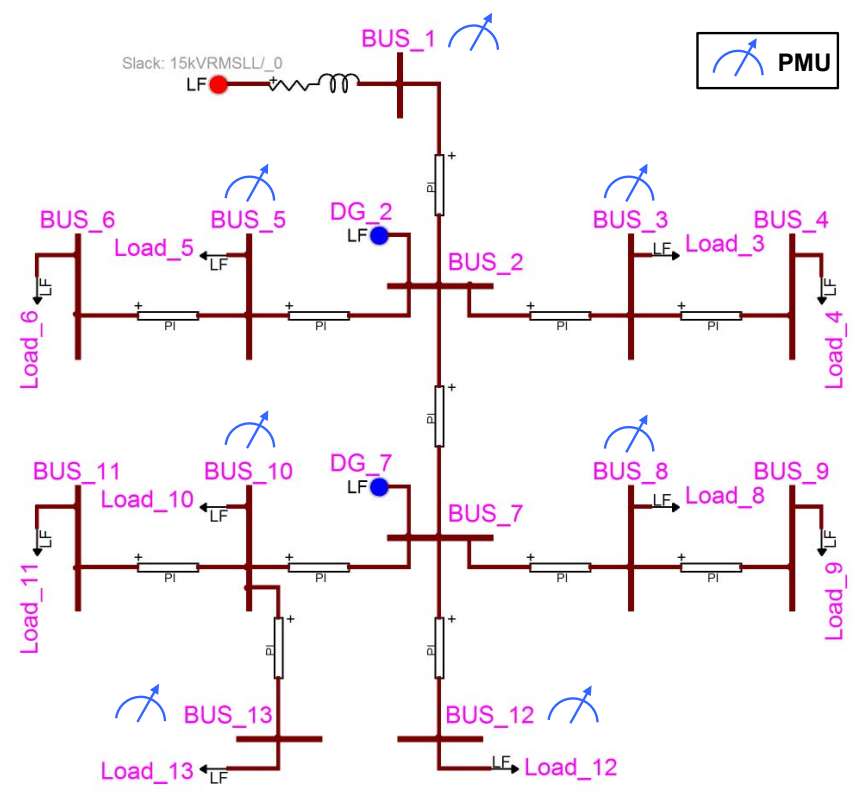

Fig. 1. The simulated IEEE 13-bus distribution test feeder.

The procedure adopted to perform the SE of the abovedescribed network is the following:

1) For each time step, i.e. every $20 \mathrm{~ms}$, a load flow is computed in order to determine the true state of the network and the ideal measurement data.

2) The measurements forwarded to the DKF-SE are obtained by perturbing the ideal measurements of step 1) with a randomly-generated noise characterized by the standard deviation of the measurement devices. The accuracy of the measurements takes into account also the presence of sensors that in the real field are connected between the grid and the PMUs. In particular, it is assumed to use 0.1-class voltage and current sensors ${ }^{2}$.

3) Then, based on the measurements provided by step 2), the state of the system is computed by using two different methods: the DKF algorithm explained in Section II, and the LWLS of [2].

The DKF-SE has been carried out for a wide range of apriori selected values of $\mathbf{Q}$. It is worth pointing out that, at this stage, $\mathbf{Q}$ is assumed to be a diagonal matrix with all equal terms, say $Q$. Figure 3 shows the norms of the p.u. errors of the system state related to all the three phases and all network buses by using values of $Q$ in the range from $10^{-20}$ to $10^{3}$ and the $20 \mathrm{~s}$ data shown in Fig. 2. It also contains the same error norms computed for a WLS state estimator (clearly independent from $Q$ ).

It can be seen that the captioned error norms exhibits a minimum that is in the range of fifteen times lower than the error norms obtained with other values of $Q$ or with the LWLSSE. Hence, this behavior denotes a strong influence of $\mathbf{Q}$ on the SE accuracy. As it can be argued, results of Fig. 3 might change if different nodal injections/absorptions are considered. This is the reason why next Section IV elaborates on the assessment of the $\mathbf{Q}$ matrix as a function of time.

\footnotetext{
${ }^{2}$ Note that the limits of ratio and phase errors imposed by [25], [26] for 0.1 -class sensors are $10^{-3}$ in p.u. and $10^{-3}$ radians, respectively.
} 

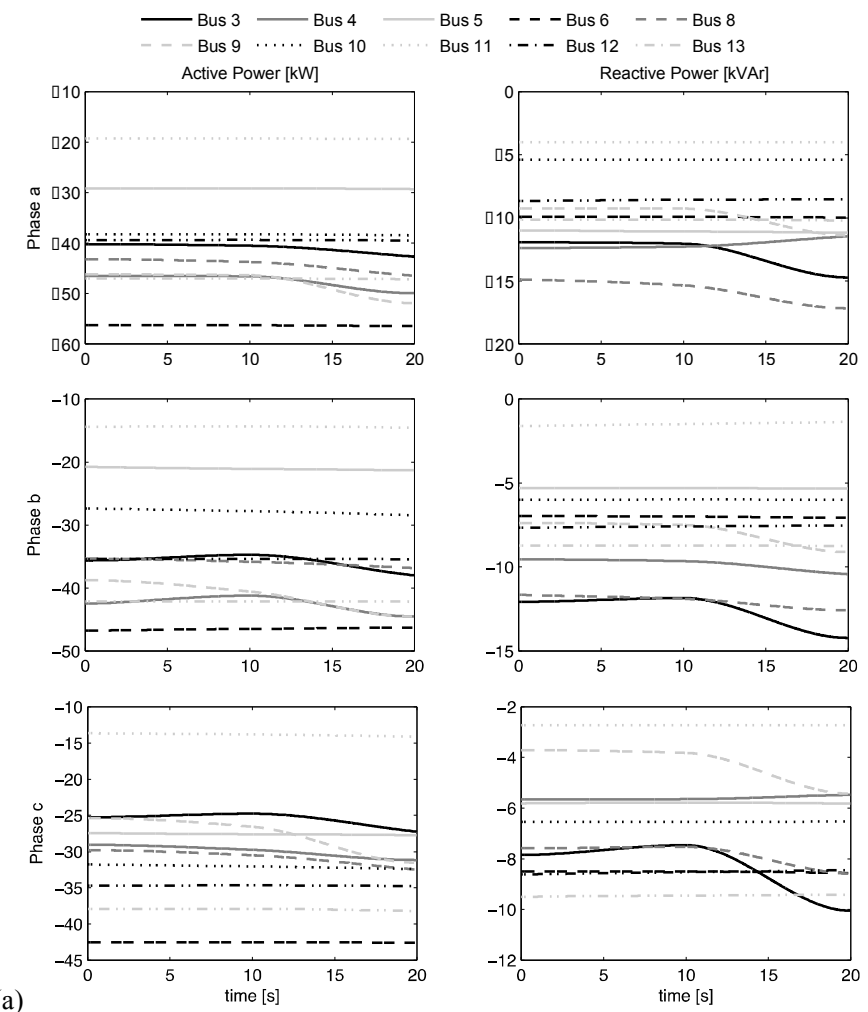

(a)
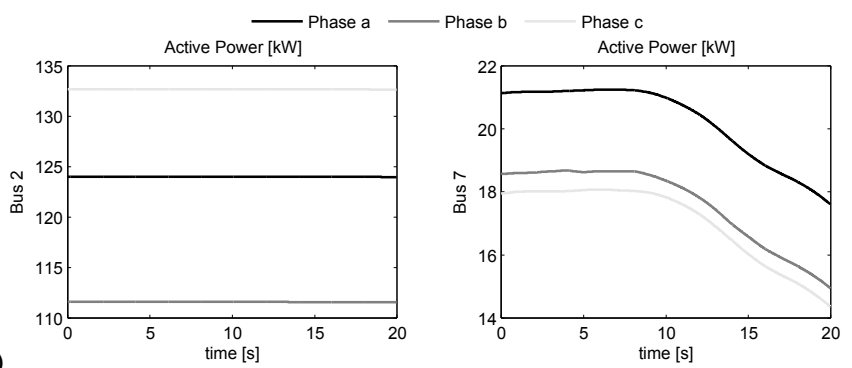

Fig. 2. Active and reactive power profiles per phase of loads and DG units used in the simulations: (a) loads, (b) DG units.
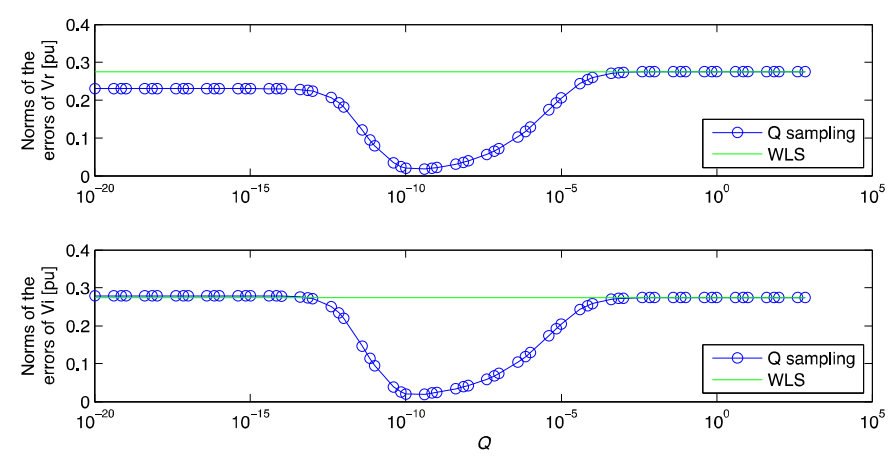

Fig. 3. Norms of the p.u. errors of the real $\left(V_{r}\right)$ and imaginary $\left(V_{i}\right)$ parts of the system state related to all the three phases and buses of the network.

\section{THE PROPOSED METHODS FOR THE ASSESSMENT OF THE OPTIMAL PROCESS NOISE COVARIANCE MATRIX}

This section presents three methods for the $\mathbf{Q}$ matrix assessment together with their numerical validation. Method 1 relies on the availability of historical probability distributions of nodal absorbed/injected power measurements that are used within a probabilistic load flow to directly infer the elements of the $\mathbf{Q}$ matrix. Method 2 and Method 3 are based on: the former on the last $N$ estimated system states available and the latter on the a-posteriori estimate error covariance matrices.

\section{A. Formulation of Method 1}

Let's assume to update the $\mathbf{Q}$ matrix every time window of a pre-determined length. Method 1 forecast $\mathbf{Q}$ for the next time window assuming the perfect knowledge of the probability distributions of the powers absorbed/injected by loads/DGs.

Being at time step $k$, the aim is to forecast $\mathbf{Q}$ for the next $M$ time steps, corresponding to a time window $T=t_{k+M}-t_{k}$. The procedure is the following:

1) Based on the assumption of the knowledge of the absorbed/injected powers in $T$, it is possible to compute the distributions, namely means $\mu$ and standard deviations $\sigma$, of the active $P_{a}$ and reactive $Q_{r}$ powers in the three phases of every node. The distributions are assumed to be uncorrelated.

$$
\begin{aligned}
& \mu_{T}=\left[\mu_{T}^{P_{a}, 1 a}, \mu_{T}^{Q_{r}, 1 a}, \mu_{T}^{P_{a}, 1 b}, \mu_{T}^{Q_{r}, 1 b}, \mu_{T}^{P_{a}, 1 c}, \mu_{T}^{Q_{r}, 1 c}, \ldots, \mu_{T}^{Q_{r}, n c}\right] \\
& \sigma_{T}=\left[\sigma_{T}^{P_{a}, 1 a}, \sigma_{T}^{Q_{r}, 1 a}, \sigma_{T}^{P_{a}, 1 b}, \sigma_{T}^{Q_{r}, 1 b}, \sigma_{T}^{P_{a}, 1 c}, \sigma_{T}^{Q_{r}, 1 c}, \ldots, \sigma_{T}^{Q_{r}, n c}\right]
\end{aligned}
$$

2) This step describes the iteration $j$ of a probabilistic load flow using a Monte Carlo simulation $\left(j=1, \ldots, N_{i t e r}\right)$.

i) The power values in the three phases of every node are randomly-generated from the distributions (9) assumed to be normal:

$$
\mathbf{S}^{(j)}=\left[P_{a, 1 a}^{(j)}, Q_{r, 1 a}^{(j)}, P_{a, 1 b}^{(j)}, Q_{r, 1 b}^{(j)}, P_{a, 1 c}^{(j)}, Q_{r, 1 c}^{(j)}, \ldots, P_{a, n c}^{(j)}, Q_{r, n c}^{(j)}\right]
$$

ii) The corresponding system state $\mathbf{x}^{(j)} \in \mathfrak{R}^{s}$ is obtained by computing a load flow.

iii) The process noise $\mathbf{w}^{(j)}$ is calculated on the basis of the assumed process model shown in (4):

$$
\mathbf{w}^{(j)}=\mathbf{x}^{(j)}-\mathbf{x}_{k}
$$

3) Once the Monte Carlo simulation has been completed, it is possible to define a $s \times N_{\text {iter }}$ matrix $\mathbf{w}$, that contains the $N_{\text {iter }}$ process noises $\mathbf{w}^{(j)} \in \mathfrak{R}^{s}$. A vector $\boldsymbol{\sigma}^{2}$ can be obtained by computing the variance of every row of $\mathbf{w}$. The elements of $\boldsymbol{\sigma}^{2}$ are the estimated process noise covariances representing the diagonal elements of the process noise covariance matrix $\mathbf{Q}$.

It is worth noting that every element of $\mathbf{w}^{(j)}$ represents the process noise of the corresponding element of the state $\mathbf{x}^{(j)}$, and in general the elements of $\mathbf{w}^{(j)}$ can be different from each other. Accordingly, the diagonal elements of $\mathbf{Q}$ are not all equal.

\section{B. Numerical validation of Method 1}

The numerical validation of all the three methods has been carried out by using the scenario described in Section III. We have inferred the statistical properties defined by (9) by using the profiles of Fig. 2. Therefore, we have assumed a perfect apriori knowledge of the nodal injections. Such a hypothesis is, of course, not realistic. However, it has been here enclosed as a proof-of-concept of the overall approach proposed in the paper. Methods 2 and 3 relax such an assumption in order to propose more realistic ways to solve this problem. Concerning Method 
1 , the number of iterations $N_{\text {iter }}$ of the Monte Carlo simulation has been set equal to 1000 and the time window $T$ equal to $1 \mathrm{~s}$.

The accuracy of the SE is shown in Fig. 4 in terms of norms of the p.u. errors of the real and imaginary components of the system state related to all the three phases and buses of the network. Fig. 4 also includes a comparison with the WLS method and the a-priori selected values of $\mathbf{Q}$ shown in Fig. 3. As it can be observed, the knowledge of the probability distribution of the nodal power injections/absorptions allows to lower-bound the accuracy of the DKF-SE process.

In order to provide a more comprehensive description of the results, Fig. 5 shows the mean and standard deviations of the errors of the network states per bus and per phase with reference to the adopted DKF-SE and the WLS. The results of Fig. 4 and Fig. 5 allows to conclude that, assuming to have a good forecast of the power profiles of loads and DGs in the near future, Method 1 is able to assess an optimal value of $\mathbf{Q}$ that leads to a large improvement of the DKF-SE accuracy (in the order of $10^{-4}$ for both real and imaginary parts) compared to WLS and to a DKF-SE that uses a-priori selected values of $\mathbf{Q}$.
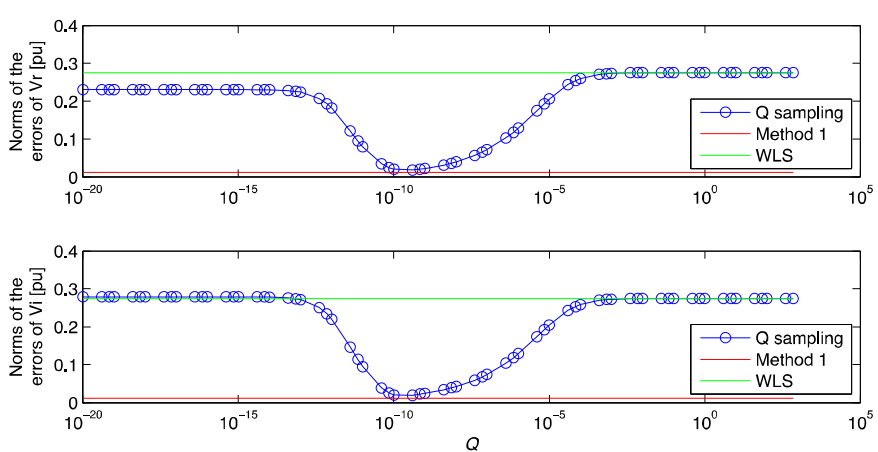

Fig. 4. Norms of the p.u. errors of the real $\left(V_{r}\right)$ and imaginary $\left(V_{i}\right)$ parts of the system state related to all the three phases and buses of the network. It shows a comparison among Method 1, WLS, and a-priori selected values of $\mathbf{Q}$
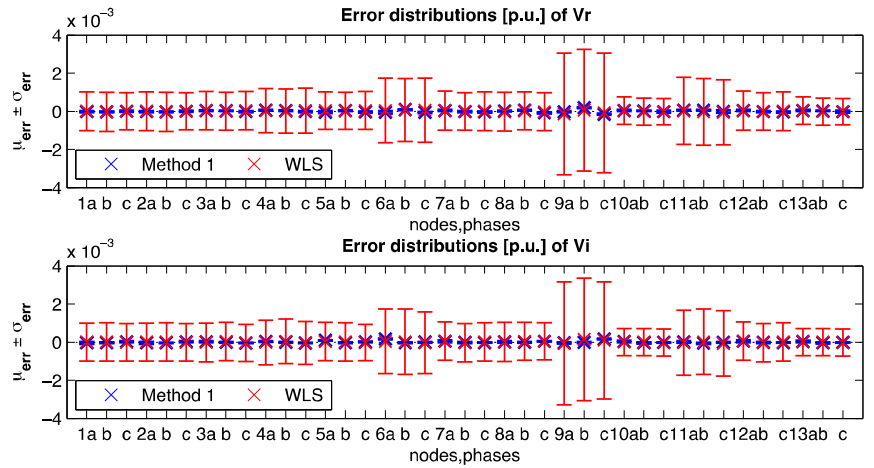

Fig. 5. Means and standard deviations of the error distributions of the real $\left(V_{r}\right)$ and imaginary $\left(V_{i}\right)$ parts of the system state per bus and per phase, with reference to Method 1 and WLS.

\section{Formulations of Methods 2 and 3}

Methods 2 and 3 do not need the hypothesis of the knowledge of what is going to happen in the future, but they are purely based on the past estimated states and on the aposteriori estimate error covariance matrices, respectively.

In principle, Method 2 is based on the fact that $\mathbf{Q}$ is not supposed to change significantly if its updating rate is high enough compared to the dynamics that are taking place in the network. In this respect, a state estimator characterized by a high refresh rate is required. Being at time step $k$, the forecast of $\mathbf{Q}$ is performed by using the last $N$ estimated states corresponding to a time window $L=t_{k}-t_{k-N}$. The procedure is explained below:

1) The $N$ process noises $\mathbf{w}^{(j)} \in \mathfrak{R}^{s}(j=1, \ldots, N)$ are computed by accounting the last $N$ estimated states available and the assumed process model shown in (4):

$$
\mathbf{w}^{(j)}=\mathbf{x}^{(j)}-\mathbf{x}_{k-N}
$$

2) We infer the process noise covariance matrix $\mathbf{Q}$ with the same procedure of step 3) of Method 1.

Regarding Method 3, it updates $\mathbf{Q}$ every time step as the uncertainty of the last SE residuals are:

$$
\sigma^{2}\left(\mathbf{r}_{k-1}\right)=\sigma^{2}\left(\mathbf{x}_{k-1}-\mathbf{x}_{k-2}\right)=\sigma^{2}\left(\mathbf{x}_{k-1}\right)+\sigma^{2}\left(\mathbf{x}_{k-2}\right)
$$

As known in KF theory, the variance of the estimated state at time step $k$ is expressed by the diagonal elements of the aposteriori estimate error covariance matrix $\mathbf{P}_{k}$.

While Method 1 is the proof of concept of the proposed approach, Method 2 and 3 provides a procedure that can be implemented in the real field where the perfect knowledge of the power distributions is unknown. It is worth noting that, opposite to Method 1, Methods 2 and 3 do not require any statistical simulation. This characteristic allows decreasing dramatically the computational overhead needed to assess the Q matrix.
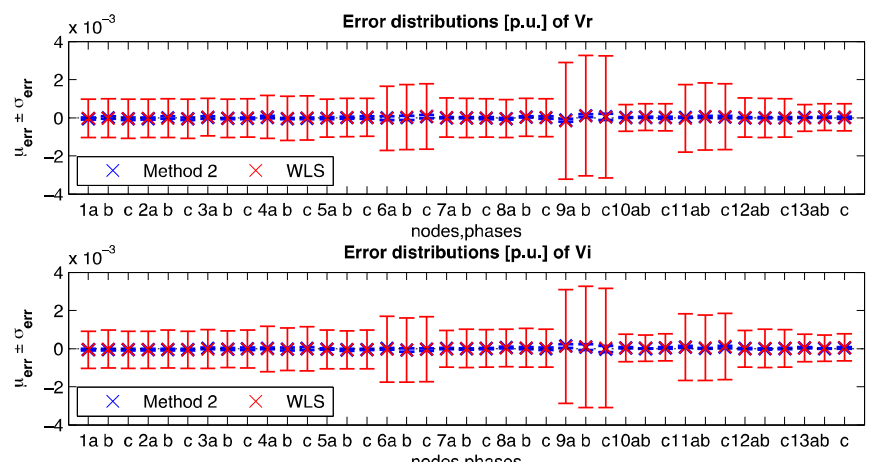

Fig. 6. Means and standard deviations of the error distributions of the real $\left(V_{r}\right)$ and imaginary $\left(V_{i}\right)$ parts of the system state per bus and per phase, with reference to Method 2 and WLS.
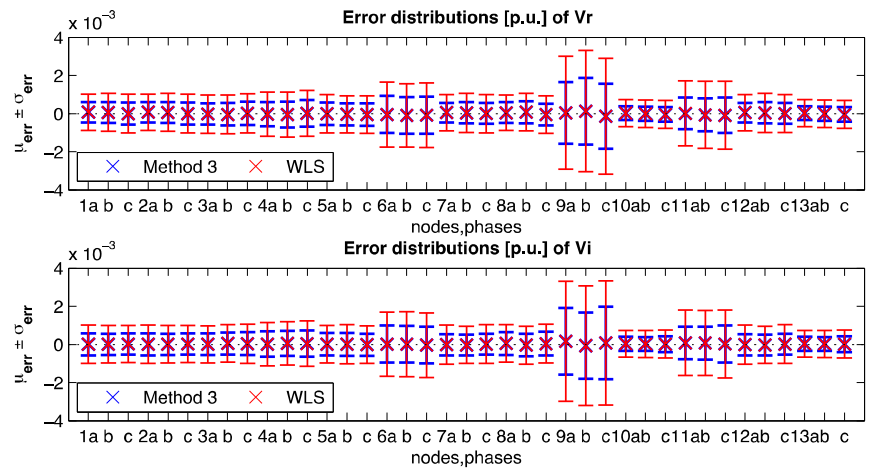

Fig. 7. Means and standard deviations of the error distributions of the real $\left(V_{r}\right)$ and imaginary $\left(V_{i}\right)$ parts of the system state per bus and per phase, with reference to Method 3 and WLS. 


\section{Numerical validation of Method 2 and 3}

This paragraph shows the SE performances achieved by applying Method 2 and 3 using the same scenario of Method 1 . For Method 2, the parameter $N$ has been set equal to 10 . The accuracy of the SE achieved with Method 2 is shown in Fig. 6 with respect to the accuracy of WLS. It can be seen that it is comparable with the accuracy achieved by Method 1 .

Moreover, Fig. 7 shows the SE accuracy reached by using Method 3. This last method allows inferring states with accuracy still better than WLS, but significantly worse compared to the accuracy obtained with Method 2. This is due to the fact that the process noise covariances inferred with Method 3 are bigger, so the DKF algorithm trusts less the adopted ARIMA $(0,1,0)$ process model. As a consequence the standard deviations of the estimation errors increase on account of the noise of the measurements.

\section{CONCLUSIONS}

Within the context of SE of ADNs, the paper has discussed the influence and the optimal assessment of the process noise covariance matrix $(\mathbf{Q})$ on the accuracy of DKF-SE processes using synchrophasors data.

The paper has first shown that error norms of the DKF-SE estimated states can exhibit a minimum that is in the range of one order of magnitude lower than error norms obtained with other values of the $\mathbf{Q}$ matrix or with the WLS-SE. Since this influence changes if different nodal injections/absorption are considered, the paper has proposed three methods for the optimal assessment of the $\mathbf{Q}$ matrix as a function of time. The first method relies on the knowledge of the probabilistic behavior of nodal network injections/absorptions and has been presented as a proof-of-concept of the proposed approach. On the other hands, second and third methods, formally similar to the first one, use the a-posteriori knowledge of the estimated states. Their impact on the accuracy improvement of the DKFSE process has been shown to be major and, in view of their low computational overhead, they are suitable for SE applications with important real-time constraints.

\section{REFERENCES}

[1] M. Paolone, M. Pignati, P. Romano, S. Sarri, L. Zanni, R. Cherkaoui, “A Hardware-in-the-loop test platform for the real-time state estimation of active distribution networks using phasor measurement units," Cigré SC6 Colloquium, 2013.

[2] K. D. Jones, J. S. Thorp, and R. Gardner, "Three-phase linear state estimation using phasor measurements," in Power and Energy Society General Meeting (PES), 2013 IEEE, 2013, pp. 1-5.

[3] "IEEE Standard for Synchrophasor Measurements for Power Systems," IEEE Standard C37.118.1-2011.

[4] "IEEE Standard for Synchrophasor Data Transfer for Power Systems," IEEE Std C37.118.2-2011 (Revision of IEEE Std C37.118-2005), pp.1$53,2011$.

[5] IEEE Guide for Design, Operation, and Integration of Distributed Resource Island Systems with Electric Power Systems, IEEE Standard $1547.4,2011$.
[6] IEEE Recommended Practice for Interconnecting Distributed Resources with Electric Power Systems Distribution Secondary Networks, IEEE Standard 1547.6, 2011.

[7] IEEE Guide for Smart Grid Interoperability of Energy Technology and Information Technology Operation with the Electric Power System (EPS), End-Use Applications, and Loads, IEEE Standard 2030, 2011.

[8] Development and Operation of Active Distribution Networks, Cigré Working Group C6.11, April 2011.

[9] K. Christakou, J.-Y. Le Boudec, M. Paolone, D.-C. Tomozei, "Efficient computation of sensitivity coefficients of node voltages and line currents in unbalanced radial electrical distribution networks," IEEE Trans. on Smart Grids, 2013.

[10]Q. Zhou and J. Bialek, "Simplified calculation of voltage and loss sensitivity factors in distribution networks," in Proc. 16th Power Syst. Comput. Conf. (PSCC2008), Glasgow, U.K., 2008.

[11]R. E. Kalman, "A new approach to linear filtering and prediction problems," Transaction of the ASME-Journal of Basic Engineering, pp. 33-45, 1960.

[12]G. Welch and G. Bishop, "An introduction to the Kalman filter," TR 95041, Dep. of Computer Science, University of North Carolina, USA, July 2006.

[13]J. Zhang, G. Welch, G. Bishop, and Z. Huang, "A two-stage Kalman filtering approach for robust and real-time power systems state tracking," IEEE Trans. on Sustainable Energy, 2013.

[14]A. Blood, M. D. Ilic, J. Ilic, and B. H. Krogh, “A Kalman filter approach to quasi-static state estimation in electric power systems," in Proc. of the 38th North American Power Symposium, 2006 (NAPS 2006), 17-19 Sept. 2006, Carbondale, IL, USA, pp. 417-422.

[15]E. A. Blood, B. H. Krogh, and M. D. Ilic, "Electric power system static state estimation through Kalman filtering and load forecasting," in Proc. of the IEEE PES General Meeting-Conversion and Delivery of Electrical Energy in the 21 st Century, Pittsburgh, PA, USA, Hawaii, USA, July 20 24, 2008, pp. 1-6.

[16]G. Valverde, V. Terzija, "Unscented kalman filter for power system dynamic state estimation," IET Generation, Transmission \& Distribution, vol. 5, No 1, pp. 29 - 37, 2011.

[17]S. Sarri, M. Paolone, R. Cherkaoui, A. Borghetti, F. Napolitano, C.A Nucci, "State estimation of active distribution networks: comparison between WLS and iterated Kalman-filter algorithm integrating PMUs," Innovative Smart Grid Technologies (ISGT Europe), 2012 3rd IEEE PES International Conference and Exhibition on. IEEE, 2012.

[18]A. Abur, and A. G. Expósito, Power System State Estimation-Theory and Implementation, New York: CRC Press, M. Dekker, 2004.

[19]S. Kosanam and D. Simon, "Kalman filtering for uncertain noise covariances," Diss. Cleveland State University, 2004.

[20]J. Zhang, G. Welch, G. Bishop, and Z. Huang, "Reduced measurementspace dynamic state estimation (ReMeDySE) for power systems," IEEE Trondheim PowerTech, 2011, pp. 1-7.

[21]A. Jain and N. R. Shivakumar, "Power system tracking and dynamic state estimation,” Power Syst. Conf. Expo., 2009, IEEE/PES, pp. 1-8.

[22]J. Dunik, M. Simandl, and O. Straka, "Methods for estimating state and measurement noise covariance matrices: aspects and comparison," System Identification. Vol. 15. No. 1. 2009.

[23]D. Efimov, A. Zolghadri, and P. Simon, "Improving fault detection abilities of extended Kalman filters by covariance matrices adjustment," Control and Fault-Tolerant Systems (SysTol), 2010 Conference on. IEEE, 2010.

[24]IEEE Distribution Planning Working Group, "Radial distribution test feeders," IEEE Trans. Power Syst., vol. 6, pp. 975-985,1991.

[25] Standard, I. E. C. 60044-1, "Instrument transformers-Part 1: Current voltage transformers", Geneva (Switzerland), 1996.

[26] Standard, I. E. C. 60044-2, "Instrument transformers-Part 2: Inductive voltage transformers", Geneva (Switzerland), 1997. 